\title{
Development of the Korean Form of the Premonitory Urge for Tics Scale: A Reliability and Validity Study
}

\author{
Mira Kim¹, Sang-Keun Chung ${ }^{1,2}$, Jong-Chul Yang ${ }^{1,2}$, \\ Jong-II Park ${ }^{1,2}$, Seok Hyun Nam ${ }^{1}$, and Tae Won Park ${ }^{1,2}$ \\ ${ }^{1}$ Department of Psychiatry, Jeonbuk National University Hospital, Jeonju, Korea \\ ${ }^{2}$ Department of Psychiatry, Jeonbuk National University Medical School, Jeonju, Korea
}

\begin{abstract}
Objectives: This study aimed to evaluate the reliability and validity of the Korean Form of the Premonitory Urge for Tics Scale (K-PUTS). Methods: Thirty-eight patients with Tourette's disorder who visited Jeonbuk National University Hospital were assessed with the K-PUTS. Together with the PUTS, the Yale Global Tic Severity Scale (YGTSS), the Children's Yale-Brown Obsessive Compulsive Scale (CY-BOCS), the attention-deficit/hyperactivity disorder (ADHD) rating scale (ARS), and the Adult ADHD Self-Report Scale (ASRS) were implemented to evaluate concurrent and discriminant validity.

Results: The internal consistency of items on the PUTS was high, with a Cronbach's $\alpha$ of 0.79 . The test-retest reliability of the PUTS, which was administered at 2 weeks to 2 months intervals, showed high reliability with a Pearson correlation coefficient of 0.60 . There was a significant positive correlation between the overall PUTS score and the YGTSS score, showing concurrent validity. There was no correlation between the PUTS, CY-BOCS, and ASRS scores, demonstrating the discriminant validity of the PUTS. Factor analysis for construct validity revealed three factors: "presumed functional relationship between the tic and the urge to tic," "the quality of the premonitory urge," and "just right phenomena."
\end{abstract}

Conclusion: The results of this study indicate that the K-PUTS is a reliable and valid scale for rating premonitory urge of tics.

Key Words: Tic; Tourette's disorder; Premonitory Urge for Tics Scale; Reliability; Validity.

Received: March 18, 2020 / Revision: March 30, 2020 / Accepted: April 16, 2020

Address for correspondence: Tae Won Park, Department of Psychiatry, Jeonbuk National University Medical School, 20 Geonji-ro, Deokjin-gu, Jeonju 54907, Korea

Tel: +82-63-250-2028, Fax: +82-63-275-3157, E-mail: ptaewon@jbnu.ac.kr

\section{INTRODUCTION}

A tic is defined as a sudden, rapid, recurrent, nonrhythmic movement or vocalization that can happen anywhere in the body [1]. Diagnostic criteria for Tourette's disorder (also known as Tourette's syndrome or Tourette syndrome) are: multiple motor tics and one or more vocal tics appearing during the course of disease; persistent tics lasting at least 1 year since first tic onset; tics waxing and waning in frequency; onset before age 18; and tics are not due to the direct physiological effects of a substance or other medical conditions [1]. Tourette's disorder usually appears at 3-8 years of age. Tic severity peaks between 10 and 12 years of age and gradually declines after puberty. Tic symptoms are substantially reduced or disappear in $60-80 \%$ of patients as they enter adulthood $[2,3]$. The most common comorbidities of Tourette's disorder are attention-deficit/hyperactivity disorder (ADHD) and obsessive-

This is an Open Access article distributed under the terms of the Creative Commons Attribution Non-Commercial License (https://creativecommons.org/licenses/by-nc/4.0) which permits unrestricted non-commercial use, distribution, and reproduction in any medium, provided the original work is properly cited. compulsive disorder (OCD). Treatment options consist mainly of behavioral therapy and medication, but it is difficult to evaluate their therapeutic effects because tics wax and wane throughout the course of disease [4].

Patients with tic disorders complain of various uncomfortable feelings or sensations before they have tic symptoms. These sensations preceding tics are termed premonitory urges. Early studies, which narrowly defined premonitory urges as "localized muscle tension," noted that only a small portion of patients with Tourette's disorder had premonitory urges $[5,6]$. Later studies reported that premonitory urges can be localized or felt systemically anywhere in the human body, and primarily in the face, neck, shoulders, arms, palms, or midline abdomen $[7,8]$. Symptoms of premonitory urges exist on a very large spectrum, with patients reporting sensations such as an excessive amount of energy, a feeling similar to hiccups, strong or intense feelings, pressure inside the brain or body, itching, an urge to move, an impulse to manifest tics, tension, a feeling that something is just not right, a sense of incompleteness, or a feeling that they will be satisfied after the 
expression of tics [8,9]. In particular, a feeling that something is just not right, the so-called "just right" phenomenon, involves all sensory perceptions including visual, tactile, and auditory sensations, and tics continue until the patient feels that "something is complete." [9] If premonitory urges are defined in the broad sense of the word to include all these symptoms, $90 \%$ of all patients with Tourette's disorder aged 10 and older are known to experience premonitory urges [10].

Very often, awareness of a premonitory urge itself causes patients to engage in tic behavior, and they feel that the urge is transiently relieved by the tic expression [10-12]. In this regard, tic disorders can be understood as the execution of a tic behavior to remove the feeling of discomfort due to a premonitory urge. Indeed, tics can be semi-voluntary (vs. involuntary) movement disorders performed with a certain intention to relieve the premonitory urge [8]. Given the therapeutic significance of premonitory urges experienced by most patients with tic disorders, it is crucial to evaluate premonitory urges preceding tics and analyze how they are associated with each other. Woods et al. [13] developed the Premonitory Urge for Tics Scale (PUTS) to measure premonitory urges. The PUTS is a self-report measure to assess the severity of premonitory urges in patients with chronic tic disorders or Tourette's disorder. The relationship between premonitory urges and tics was investigated by examining the relationship between the PUTS score and the individual items of the Yale Global Tic Severity Scale (YGTSS). The association between premonitory urges and other psychiatric symptoms was investigated by comparing the PUTS with specific items of the Child Behavior Checklist (CBCL) and Children's Yale-Brown Obsessive-Compulsive Scale (CY-BOCS). Whereas the PUTS was found to be stable with a high internal consistency and demonstrated its concurrent and discriminant validity when compared with YGTSS in the age group of 10 and over, neither internal consistency nor concurrent validity could be confirmed in the younger group ( $<10$ years). Consequently, the PUTS was recommended for children aged 10 and older [13]. In a study with youth aged 9 to 17 , Rozenman et al. [14] reported that the intensity of anxiety experienced by patients with tic disorders and the severity of somatic/panic symptoms measured by the Screen for Child Anxiety Related Emotional Disorders-Child Version questionnaire (SCARED-C) were associated with the PUTS total score. If this association can be analyzed for individual children affected, these results can be used to control the severity of premonitory urges in behavioral therapy involving classical conditioning [4]. As examined above, accurate understanding and assessments of premonitory urges is essential for the diagnosis and treatment of tic disorders. However, the reliability and validity of the PUTS have yet to be tested in South Korea.
Therefore, this study was conducted to evaluate the reliability and validity of the Korean Form of the PUTS developed by Woods et al. [13].

\section{METHODS}

\section{Subjects}

The subjects of this study were patients who had presented to the psychiatric outpatient clinic of Jeonbuk National University Hospital from July 2017 to July 2019. Subjects recruited were aged 10 and older, had been diagnosed with Tourette's disorder or chronic tic disorders based on an interview with a psychiatrist using DSM-5 criteria, and showed premonitory urges in a pre-interview. Patients with developmental disorders (intellectual disability, language impairments, autistic spectrum disorder, etc.), major psychiatric disorders (schizophrenia, bipolar disorder, etc.), and major medical and neurological disorders were excluded. Thirty-eight children, adolescents, and adults were enrolled in the study. The mean age of the subjects ( 31 males and 7 females) was 15.5 years (range: $10-44)$, and no gender-dependent age difference was observed (mean age of males: $16.96 \pm 7.87$ years, mean age of females: $15.57 \pm 6.05$ years; $t=0.44, p>0.05$ ). The subjects were divided into two age groups: $10-15$ years (primary and middle school students) and $\geq 16$ years (high school students and adults). No significant inter-group difference was observed in gender distribution, with 17 males (80.96\%) and 4 females (19.04\%) in the younger group (10-15 years), and 14 males (82.36\%) and 3 females (17.64\%) in the older group ( $\geq 16$ years). With regard to co-occurring diseases, ADHD was most frequently observed ( $\mathrm{n}=13,34.21 \%)$, followed by major depressive disor$\operatorname{der}(\mathrm{n}=4,10.52 \%), \mathrm{OCD}(\mathrm{n}=1,2.63 \%)$, social anxiety disorder $(\mathrm{n}=1,2.63 \%)$, and panic disorder $(\mathrm{n}=1,2.63 \%)$. This study was approved by the Institutional Review Board (IRB) of Jeonbuk National University Hospital (IRB No. CUH 2017-02-017).

\section{Measures}

\section{Premonitory Urge for Tics Scale (PUTS)}

In this study, the Korean Form of the PUTS was used by adapting the original PUTS presented by Woods et al. [13] in 2005. The PUTS was evaluated in two stages. First, 10 items were formulated based on the phenomenological descriptions of premonitory urges presented in the literature. Second, after excluding Item 10 based on feedback on the words in individual items and content of the scale provided by a panel of experts in Tourette's disorder treatment and research, the final scale comprised 9 items. The reliability and validity of these items were assessed on the original English version.

Each of the 9 items of the PUTS, a self-report questionnaire, 
is rated on a 4-point scale ranging from 1 (not at all true) to 4 (very much true). That is, the total score ranges from 9 to 36 points, with a higher score indicating a stronger premonitory urge [15].

The Korean Form of the PUTS was produced as follows: The PUTS was translated by two pediatric psychiatrists at Jeonbuk National University Hospital, and the Korean translation was back-translated into English by two medical students with native-level English proficiency. The back-translation was reviewed by Douglas W. Woods, the author of the original PUTS version, and compared with the original English version. The Korean version was corrected and finalized based on the comparison feedback from the original author.

\section{Korean Form of Yale Global Tic Severity Scale (YGTSS)}

The Korean Form of YGTSS was used in this study to investigate the concurrent validity of the PUTS [16]. This measure is divided into home-assessed and clinician-rated measures. In this study, the clinician-rated measure was used in which a clinician directly assesses tic severity of each subject. The YGTSS clinician-rated measure is designed to assess the tic severity based on a home assessment questionnaire (selfreport) and the results of a semi-structured interview. Based on the tic symptoms checklist, the clinician rates the motor and vocal tics in five dimensions: 1) number, 2) frequency, 3) intensity, 4) complexity, and 5) interference. Each of these dimensions is rated on a 6-point scale. Impairment is separately rated irrespective of tic types, with the main focus on the effect of the tic disorders over the last week on the person affected in terms of self-esteem, family life, social acceptance (peer relationship), or school or job functioning. The total tic severity score (YGTSS total score) is calculated as the sum of the motor tic severity and the vocal tic severity in this study [13]. The validity and reliability of this measure have been adequately demonstrated in many studies [17].

\section{Korean Form of Yale-Brown Obsessive-Compulsive Scale (Y-BOCS) and Korean Form of Children's Yale-Brown Obsessive-Compulsive Scale (CY-BOCS)}

To investigate the discriminant validity of the PUTS with regard to OCD, the age-dependent Korean Forms of Y-BOCS and CY-BOCS were used. The CY-BOCS for youth up to age 18 who are diagnosed with OCD is the children's version of the Y-BOCS for adults [18], which is a 10-item semi-structured clinician-rated instrument for rating OCD severity over the past week, and is rated in the same manner as the Y-BOCS. The overall structure, assessment criteria, and rating scales are the same as the Y-BOCS, but the questions about symptoms are modified in terms of form and expression to reflect the developmental stage of children. The rater is instructed to make a final judgment based on the information obtained from both children and parents, recording the most severe obsessive-compulsive thoughts and behaviors as the target symptoms after completing the overall rating of the symptom checklist. The severity of a target symptom is rated by asking: 1) time occupied by obsessive thoughts, 2) interference due to obsessive thoughts, 3) distress associated with obsessive thoughts, 4) resistance against obsessions, and 5) degree of control over obsessive thoughts. Each item is rated on a 5point scale [19]. The validity and reliability of this measure have been well-demonstrated in many studies [20].

\section{ADHD Rating Scale (ARS)}

The ADHD Rating Scale (ARS) was developed by DuPaul [21] as a measure of behaviors observed in school-aged children with ADHD. This measure was used in this study to examine the discriminant validity of the PUTS. The ARS is composed of 18 items matching the ADHD diagnostic criteria specified in the DSM-IV. Each item is rated on a 4-point scale from 0 to 3 points depending on the severity of ADHD symptoms. The ARS lends itself well to distinguishing children with learning disabilities from normal children and children with ADHD from children without ADHD [22]. The validity and reliability of this measure have been sufficiently demonstrated in many studies [21,23].

\section{Adult ADHD Self-Report Scale (ASRS)}

The Adult ADHD Self-Report Scale (ASRS) is an 18-item psychometric instrument developed under the aegis of the World Health Organization (WHO) [24-26]. The screening tool (Part A) of this scale consists of 6 items designed to measure only the frequency of symptoms. Though originally designed for adults ( $\geq 18$ years), its applicability to adolescents was demonstrated in a study [27]. In this study, a 5-point scale ( 1 to 5 ) was used to rate severity (score range: 18 to 90 points).

\section{Statistical analysis}

Statistical analysis was performed using SPSS software (Version 18.0, SPSS Inc., Chicago, IL, USA).

\section{Reliability}

\section{Internal consistency}

Internal consistency was estimated using Cronbach's alpha for the PUTS scores of subjects $(n=38)$ and calculating the Pearson correlation coefficients between each item score and the total score.

Test-retest reliability

The subjects were administered the PUTS twice (intervals 
ranged from 2 weeks to 2 months), and the test-retest reliability was estimated with the Pearson correlation coefficient.

\section{Validity}

Concurrent validity

Concurrent validity was assessed in two steps: first, computation of the Pearson correlation coefficient between the PUTS and the YGTSS in 38 subjects; second, computation of Spearman's correlation coefficients between the PUTS total score and the YGTSS subscale scores (motor tic severity, vocal tic severity, and impairment) in 20 subjects.

\section{Discriminant validity}

The discriminant validity of the PUTS was tested by comparing it with other tools for measuring behavioral dimensions, namely the CY-BOCS (10-17 years), the Y-BOCS $(\geq 18$ years), the ARS (10-15 years), and the ASRS ( $\geq 16$ years). The Pearson correlation coefficients were computed between the total score of each of these measures and the total score of the PUTS as well as the scores of individual items.

\section{Construct validity}

A principal axis factor analysis was performed on the 9 items of the PUTS to identify its inner structure. Three factors with an eigenvalue of 1 or over were extracted, and the factor structure was examined with a varimax rotation.

\section{RESULTS}

\section{Results of evaluation with the PUTS and other measures}

The mean PUTS scores at initial administration and retest were $21.28 \pm 5.87$ and $21.15 \pm 7.78$ points, respectively. No statistically significant differences in the mean PUTS score were observed between the two age groups; mean scores were 21.23 \pm 5.90 in the younger group (10 to 15 years) and $21.35 \pm 6.00$ for the older group ( $\geq 16$ years). The reason for using 16 as the upper threshold age is that the PUTS was initially tested with youths aged 8-16 [13] and subsequently with adults aged 1669 by the original author [15]. Furthermore, as mentioned above, 16 is the threshold age between the ARS and ASRS which were employed to test the discriminant validity.

The mean YGTSS motor tic severity, vocal tic severity, and

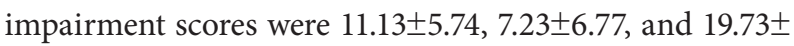
11.50, respectively, yielding a total YGTSS mean score of 38.10 \pm 20.36 . The mean scores of the CY-BOCS, Y-BOCS, ARS, and ASRS were 15.73 $\pm 9.90,15.44 \pm 9.98,10.04 \pm 8.62$, and $31.71 \pm 12.59$, respectively (Table 1 ).

\section{Reliability verification}

\section{Internal consistency}

The internal consistency of the 9 items of the PUTS was considered good, with a Cronbach's alpha of 0.79 .

\section{Test-retest reliability}

The test-retest reliability of the PUTS over a time interval of 2 weeks to 2 months was high, with a Pearson correlation coefficient of 0.60 .

\section{Validity verification}

\section{Concurrent validity}

Pearson correlation analysis was performed to identify the correlations between the total PUTS score and the YGTSS subscale scores (motor tic severity, vocal tic severity, and impairment) and YGTSS total score. The analysis revealed significant positive correlations between the first total PUTS scores and YGTSS total scores $(\mathrm{r}=0.55, \mathrm{p}<0.001)$, YGTSS motor tic $(\mathrm{r}=0.48, \mathrm{p}<0.01)$, YGTSS vocal tic $(\mathrm{r}=0.51, \mathrm{p}<0.01)$, and YGTSS impairment score $(\mathrm{r}=0.43, \mathrm{p}<0.01)(\mathrm{n}=38)$ (Table 2).

Spearman's correlation analysis was then performed on the second total PUTS score $(\mathrm{n}=20$ after excluding missing data). The analysis revealed significant positive correlations between the second total PUTS score and the YGTSS total score $(\mathrm{r}=0.65, \mathrm{p}<0.01)$, YGTSS motor tic severity $(\mathrm{r}=0.66, \mathrm{p}<$ $0.01)$, YGTSS vocal tic severity $(\mathrm{r}=0.57, \mathrm{p}<0.01)$, and YGTSS impairment score $(\mathrm{r}=0.63, \mathrm{p}<0.01)(\mathrm{n}=20)$ (Table 2).

The total PUTS score also showed a significant positive correlation with each dimension of the YGTSS: 1) number, 2) frequency, 3) intensity, 4) complexity, and 5) interference (Table 3).

Table 1. Summary of clinical assessment

\begin{tabular}{lrrcc}
\hline \multicolumn{1}{c}{ Scale } & Mean & SD & Range & Remarks (n) \\
\hline PUTS 1st & 21.28 & 5.87 & $9-31$ & 38 \\
PUTS 2nd & 21.15 & 7.78 & $9-33$ & 20 \\
YGTSS motor tics & 11.13 & 5.74 & $0-24$ & 38 \\
YGTSS phonic tics & 7.23 & 6.77 & $0-24$ & 38 \\
YGTSS total & 18.37 & 10.96 & $3-48$ & 38 \\
YGTSS impairment & 19.73 & 11.50 & $0-40$ & 38 \\
CY-BOCS & 15.73 & 9.90 & $6-25$ & 11 \\
Y-BOCS & 15.44 & 9.98 & $0-35$ & 9 \\
ARS & 10.04 & 8.62 & $0-26$ & 21 \\
ASRS & 31.71 & 12.59 & $18-54$ & 14 \\
\hline
\end{tabular}

PUTS: Premonitory Urge to Tics Scale, YGTSS: Yale Global Tic Severity Scale, YGTSS total: YGTSS motor tics+YGTSS phonic tics, CYBOCS: Children's Yale-Brown Obsessive Compulsive Scale, Y-BOCS: Yale-Brown Obsessive Compulsive Scale, ARS: ADHD (attentiondeficit/hyperactivity disorder) Rating Scale, ASRS: Adult ADHD Self Report Scale, SD: standard deviation 


\section{Discriminant validity}

For verification of discriminant validity, Spearman's correlation analysis was performed to assess the correlations between the total PUTS score and the subscale scores (obsessive-compulsive thoughts and behaviors) and total scores of the Y-BOCS and CY-BOCS $(\mathrm{n}=20)$. As a result, no significant correlations were observed between the total PUTS score and any of them.

Spearman's correlation analysis was also used to identify the correlations between the total scores of the PUTS, ARS, and ASRS for the younger group $(\mathrm{n}=21)$ and the older group $(\mathrm{n}=14)$, respectively. As a result, a negative correlation was observed between the PUTS and the ARS $(r=-0.47, \mathrm{p}<0.05)$ and no significant correlation was confirmed between the PUTS and the ASRS.

\section{Construct validity}

A factor analysis was performed on the 9 items of the PUTS to identify its latent structure. As a factor extraction method, the principal axis factor analysis was used along with a varimax rotation.

The Kaiser-Meyer-Olkin (KMO) value obtained was 0.69 , which indicates that the factor analysis model is appropriate for the data. Bartlett's test of sphericity also yielded a significant result indicating the suitability of the factor analysis model $(p<0.05)$. With a cumulative variance of $56.94 \%$, three underlying factors were found to have high explanatory power.

The first factor contained four items $(1,7,8,9)$, the second factor three items $(2,3,6)$, and the third factor two items $(4,5)$. Based on the properties of the constituent items, the first factor was named the "presumed functional relationship between the tic and the urge to tic," the second factor "the quality of the premonitory urge," and the third factor "just right phenomena." All factor loadings $(\geq 0.4)$ met the 0.4 cutoff, the re- quirement for the validity of general psychometric instruments. These results allowed us to proceed with the factor analysis without further item removal or adjustment. These results suggest that the PUTS has high construct validity as a diagnostic measure of a premonitory urge preceding a tic (Table 4).

\section{DISCUSSION}

With a Cronbach's alpha of 0.79 for the 9 items of the PUTS, each item was found to consistently measure premonitory urges. The correlation coefficients computed in this study slightly deviate from the internal consistency yielded in the study of Woods et al. [13] who developed the PUTS. The Cronbach's alpha in Woods and colleagues' study was as high as $0.81(\mathrm{n}=42)$, with a substantial difference between the younger ( $\leq 10$ years) and older ( $\geq 11$ years) subjects at $0.57(n=19)$ and $0.89(\mathrm{n}=23)$, respectively. However, the Cronbach's alpha val-

Table 4. Principal axis factor analysis for PUTS

\begin{tabular}{|c|c|c|c|}
\hline Item & 1 & 2 & 3 \\
\hline PUTS 8 & 0.10 & 0.08 & 0.14 \\
\hline PUTS 7 & 0.74 & 0.34 & 0.08 \\
\hline PUTS 1 & 0.45 & 0.36 & -0.04 \\
\hline PUTS 9 & 0.44 & 0.11 & 0.14 \\
\hline PUTS 6 & 0.16 & 0.69 & 0.14 \\
\hline PUTS 2 & 0.13 & 0.65 & 0.14 \\
\hline PUTS 3 & 0.30 & 0.57 & 0.01 \\
\hline PUTS 4 & 0.19 & -0.01 & 0.93 \\
\hline PUTS 5 & 0.08 & 0.49 & 0.72 \\
\hline Eigen value & 1.94 & 1.71 & 1.47 \\
\hline Common variance (\%) & 21.56 & 19.00 & 16.38 \\
\hline Cumulative variance (\%) & 21.56 & 40.56 & 56.94 \\
\hline
\end{tabular}
$\mathrm{KMO}^{*}=0.69$, Bartlett's $\chi^{2 \dagger}=112.85(\mathrm{p}<0.001)$

*Kaiser-Meyer-Oklin (KMO) measure, 'Bartlett's Test of Sphericity. PUTS: Premonitory Urge to Tics Scale

Table 2. Comparison of PUTS 1st scores $(n=38)$ and PUTS 2 nd scores $(n=20)$ with concurrent clinical rating instruments

\begin{tabular}{lccccc}
\hline \multicolumn{1}{c}{ Scale } & $\begin{array}{c}\text { PUTS total } \\
\text { 1st (2nd) }\end{array}$ & $\begin{array}{c}\text { YGTSS motor tics } \\
\text { 1st (2nd })\end{array}$ & $\begin{array}{c}\text { YGTSS phonic tics } \\
\text { 1st (2nd })\end{array}$ & $\begin{array}{c}\text { YGTSS impairment } \\
\text { 1st (2nd) }\end{array}$ & $\begin{array}{c}\text { YGTSS total } \\
\text { 1st (2nd })\end{array}$ \\
\hline PUTS total 1st (2nd) & $1.00(1.00)$ & & & & \\
YGTSS motor tics 1st (2nd) & $0.48^{*}\left(0.66^{*}\right)$ & $1.00(1.00)$ & & & \\
YGTSS phonic tics 1st (2nd) & $0.51^{*}\left(0.57^{*}\right)$ & $0.52^{*}\left(0.69^{*}\right)$ & $1.00(1.00)$ & & \\
YGTSS impairment 1st (2nd) & $0.43^{*}\left(0.63^{*}\right)$ & $0.66^{\dagger}\left(0.77^{\dagger}\right)$ & $0.48^{*}\left(0.62^{*}\right)$ & $1.00(1.00)$ & $1.00(1.00)$ \\
YGTSS total 1st (2nd) & $0.57^{*}\left(0.69^{*}\right)$ & $0.85^{*}\left(0.90^{*}\right)$ & $0.90^{*}\left(0.93^{*}\right)$ & $0.64^{*}\left(0.72^{*}\right)$ &
\end{tabular}

${ }^{*} p<0.01,{ }^{\dagger} p<0.001$. PUTS: Premonitory Urge to Tics Scale, YGTSS: Yale Global Tic Severity Scale

Table 3. Correlation analysis between YGTSS scales and PUTS global score obtained at the 1st (2nd) visit for total sample

\begin{tabular}{cccccc}
\hline Variable & Number & Frequency & Intensity & Complexity & Interference \\
\hline PUTS 1st $(\mathrm{n}=38)$ & $0.55^{\dagger}$ & $0.59^{\dagger}$ & $0.53^{\dagger}$ & $0.35^{*}$ & $0.52^{\dagger}$ \\
PUTS 2nd $(\mathrm{n}=20)$ & $0.54^{*}$ & $0.60^{\dagger}$ & $0.69^{\dagger}$ & $0.54^{*}$ & $0.53^{*}$
\end{tabular}

Correlation was obtained using Pearson correlation for the 1 st visit and spearman correlation for the 2 nd visit. ${ }^{*} \mathrm{p}<0.05,{ }^{\dagger} \mathrm{p}<0.01$.

PUTS: Premonitory Urge to Tics Scale, YGTSS: Yale Global Tic Severity Scale 
ues in the current study were $0.79(\mathrm{n}=21)$ for the younger group (10-15 years) and $0.81(n=17)$ for the older group ( $\geq 16$ years), with the overall Cronbach's alpha demonstrating a stable internal consistency across the age span. This may be explained by the lower age cutoff (10 years) of the subjects in this study in accordance with Woods' recommendation.

In a study investigating the validity of the YGTSS conducted by Chung et al., [16] the total tic score included the impairment score. In this study, however, the total YGTSS score did not include the impairment score in accordance with the PUTS validity and reliability study by Woods et al. [13]. Of note, the concurrent validity was also statistically significant when the impairment score was included in the total score (data not presented).

A comparison of the total PUTS score with the YGTSS dimensions (i.e., number, frequency, intensity, complexity, and interference) revealed positive correlations between all items and thus demonstrated its concurrent validity. In the study conducted by Woods et al., [13] positive correlations were observed only in number, complexity, and interference; broken down by age group, the younger group ( $\leq 10$ years) did not show any significant correlations between the total and individual scores of the YGTSS, and the older group ( $\geq 11$ years) showed correlations between the total score and the number, complexity, and interference scores. The same paper pointed out the non-verification of concurrent validity in the younger group ( $\leq 10$ years) as a limitation, and the fact that the lower age cutoff was set at 10 years in this study is presumably the reason for the difference in the concurrent validity between this study and the study conducted by Woods et al. [13].

In the assessment of discriminant validity, the PUTS and ARS were found to have a negative correlation ( $\mathrm{r}=-0.47, \mathrm{p}<$ 0.05). The difference between the parent-rated ARS and selfreported PUTS may be ascribable to differences in rater characteristics, which is beyond the scope of this paper. In contrast, the ASRS, a self-report scale evaluating ADHD in adults $(\geq 16$ years), did not show a significant correlation with the PUTS although administered by the same raters (the subjects themselves). A low correlation with a theoretically irrelevant variable in a measure that corrects for this limitation (i.e., self-report instead of parent-rated) indicates that these measures (PUTS and ARS/ASRS) show discriminant validity as measures for different diseases.

Three factors with eigenvalues greater than or equal to 1 were extracted as a result of factor analysis performed on the 9 items of the PUTS. The first factor containing items 1, 7, 8, and 9 was named "the presumed functional relationship between the tic and the urge," bundling together all premonitory urges preceding tics and disappearing with tic expression. Item 1 ("Right before I do a tic, I feel like my insides are itchy") was also found to share a common characteristic with the second factor, "the quality of the premonitory urge" ( 0.45 vs. 0.36$)$. The second factor containing items 2,3 , and 6 was named "the quality of the premonitory urge," expressing the pressure or tension inside and uncontrollable energy felt right before tic behaviors. The third factor containing items 4 and 5 was named "just right phenomena" by bundling together the feelings of something being just not right and not complete. These results can be compared with those of previous studies as follows: In a study with patients aged 7-55, Brandt et al. [28] bundled items 1,7 , and 8 together and named it "the overall intensity of premonitory urge," items 2-5 "the quality of premonitory urge," and items 6 and 9 "the perceived control over tics and premonitory urge." The first and second factors roughly coincide with those of our study. One salient difference is that our study extracted items 4 and 5 as one factor. In a study with patients ages 16-69, Reese et al. [15] named items 1-6 "the quality of premonitory urge," items 7 and 8 "the frequency of the premonitory urge to tic," and item 9 "the presumed functional relationship between the tic and the urge to tic." What this study has in common with our study is the extraction of the factors "the presumed functional relationship between the tic and the urge to tic" and "the quality of premonitory urge." Differences may be attributable to subject characteristics including age, and linguistic nuances due to the translation process of the adaptation.

Limitations of this study include that the interval between test and retest was not regular but ranged from 2 weeks to 2 months, which may result in memory-dependent error fluctuations. Additionally, the nature of tic symptoms that can undergo abrupt changes over time makes them inherently prone to test-retest variability. Given the close association between tic disorders and premonitory urges, the latter can also be assumed to be prone to time-dependent variabilities, which lessens the importance of test-retest reliability verification. Alongside this, despite the advantage of easy evaluation as a self-report scale, the PUTS has the disadvantage of high score variability depending on the rater's attitude, motivation, and intellectual level [4]. This drawback of a self-report scale will be overcome if a clinician-administered structured rating scale is developed. Lastly, whereas the OCD comorbidity rate in patients with Tourette's disorder is known to be about 30\%, OCD comorbidity was rarely observed in this study. This suggests that the characteristics of subjects in this study were somewhat different from those of previous studies.

\section{CONCLUSION}

From the study results, it is evident that the Korean Form of the PUTS is a measure with high validity and reliability in 
evaluating the premonitory urges in patients with Tourette's disorder. Examining the role of premonitory urges in patients with Tourette's disorder and efficiently applying the results to therapy would greatly contribute to improving the patients' quality of life. The Korean from of the PUTS is expected to serve as a useful instrument for studying the epidemiology and clinical course of Tourette's disorder and its therapeutic effects.

\section{Acknowledgments}

This paper was supported by Fund of Biomedical Research Institute, Jeonbuk National University Hospital. In addition, the authors would like to thank Professor DW Woods for his efforts in review of back translation from Korean to English of Premonitory Urge for Tics Scale.

\section{Conflicts of Interest}

The authors have no potential conflicts of interest to disclose.

\section{Author Contributions}

Conceptualization: Tae Won Park. Data curation: Tae Won Park, Seok Hyun Nam. Formal analysis: Seok Hyun Nam, Mira Kim. Funding acquisition: Tae Won Park. Investigation: Tae Won Park, Mira Kim. Methodology: Tae Won Park, Mira Kim, Jong-Il Park, Sang-Keun Chung, Jong-Chul Yang. Project administration: Tae Won Park, Mira Kim. Resources: Tae Won Park, Seok Hyun Nam, Mira Kim. Software: Mira Kim. Supervision: Sang-Keun Chung, Jong-Chul Yang, Jong-Il Park. Validation: Tae Won Park. Writing_original draft: Mira Kim. Writing_-review \& editing: Tae Won Park.

\section{ORCID iDs}

Mira Kim https://orcid.org/0000-0001-6804-5369

Sang-Keun Chung https://orcid.org/0000-0002-3488-6351

Jong-Chul Yang https://orcid.org/0000-0002-7701-2718

Jong-Il Park https://orcid.org/0000-0003-4690-397X

Seok Hyun Nam https://orcid.org/0000-0003-3972-1553

Tae Won Park

\section{REFERENCES}

1) American Psychiatric Association. Diagnostic and statistical manual of mental disorders: DSM-5. 5th ed. Arlington, VA: American Psychaitric Association;2013.

2) Leckman JF, Zhang H, Vitale A, Lahnin F, Lynch K, Bondi C, et al. Course of tic severity in Tourette syndrome: the first two decades. Pediatrics 1998;102:14-19.

3) Rajagopal S, Seri And S, Cavanna AE. Premonitory urges and sensorimotor processing in Tourette syndrome. Behav Neurol 2013;27: 65-73.

4) Nam SH, Park JH, Park TW. Clinical aspect of premonitory urge in patients with Tourette's disorder. J Korean Acad Child Adolesc Psychiatry 2019;30:50-56.

5) Montgomery MA, Clayton PJ, Friedhoff AJ. Psychiatric illness in Tourette syndrome patients and first-degree relatives. Adv Neurol 1982;35:335-339.

6) Shapiro AK, Shapiro ES, Young JG, Feinberg TE. Gilles de la Tourette syndrome. New York, NY: Raven Press, Ltd;1988.

7) Miguel EC, Coffey BJ, Baer L, Savage CR, Rauch SL, Jenike MA. Phenomenology of intentional repetitive behaviors in obsessivecompulsive disorder and Tourette's disorder. J Clin Psychiatry 1995;

$56: 246-255$

8) Kwak C, Dat Vuong K, Jankovic J. Premonitory sensory phenomenon in Tourette's syndrome. Mov Disord 2003;18:1530-1533.

9) Leckman JF, Walker DE, Goodman WK, Pauls DL, Cohen DJ. "Just right" perceptions associated with compulsive behavior in Tourette's syndrome. Am J Psychiatry 1994;151:675-680.

10) Leckman JF, Walker DE, Cohen DJ. Premonitory urges in Tourette's syndrome. Am J Psychiatry 1993;150:98-102.

11) Brandt VC, Beck C, Sajin V, Baaske MK, Bäumer T, Beste C, et al. Temporal relationship between premonitory urges and tics in Gilles de la Tourette syndrome. Cortex 2016;77:24-37.

12) Seki K, Fetz EE. Gating of sensory input at spinal and cortical levels during preparation and execution of voluntary movement. J Neurosci 2012;32:890-902.

13) Woods DW, Piacentini J, Himle MB, Chang S. Premonitory Urge for Tics Scale (PUTS): initial psychometric results and examination of the premonitory urge phenomenon in youths with Tic disorders. J Dev Behav Pediatr 2005;26:397-403.

14) Rozenman M, Johnson OE, Chang SW, Woods DW, Walkup JT, Wilhelm S, et al. Relationships between premonitory urge and anxiety in youth with chronic tic disorders. Child Health Care 2015;44: 235-248.

15) Reese HE, Scahill L, Peterson AL, Crowe K, Woods DW, Piacentini J, et al. The premonitory urge to tic: measurement, characteristics, and correlates in older adolescents and adults. Behav Ther 2014;45: 177-186.

16) Chung SJ, Lee JS, Yoo TI, Koo YJ, Jeon SI, Kim BS, et al. Development of the Korean Form of Yale Global Tic Severity Scale: a validity and reliability study. J Korean Neuropsychiatr Assoc 1998; 37:942-951.

17) Leckman JF, Riddle MA, Hardin MT, Ort SI, Swartz KL, Stevenson J, et al. The Yale Global Tic Severity Scale: initial testing of a clinician-rated scale of tic severity. J Am Acad Child Adolesc Psychiatry 1989;28:566-573.

18) Goodman WK, Price LH, Rasmussen SA, Mazure C, Fleischmann RL, Hill CL, et al. The Yale-Brown Obsessive Compulsive Scale. I. Development, use, and reliability. Arch Gen Psychiatry 1989;46:10061011.

19) Chung SJ, Lee JS, Kang YH, Cho SJ, Suh DH, Hong KE. Development of Korean Form of Children's Yale-Brown Obsessive-Compulsive Scale(CY-BOCS): a reliability and validity study. J Korean Acad Child Adolesc Psychiatry 2000;11:60-69.

20) Goodman WK, Rasmussen SA, Price LH, Riddle MA, Rapoport JL. Children's Yale-Brown Obsessive-Compulsive Scale (CY-BOCS). New Haven, CT: Yale University Press; 1986.

21) DuPaul GJ. Parent and teacher rating of ADHD symptoms: psychometric properties in a community based sample. J Clin Child Psychol 1991;20:245-253.

22) So YK, Noh JS, Kim YS, Ko SK, Koh YJ. The reliability and validity of Korean Parent and Teacher ADHD Rating Scale. J Korean Neuropsychiatr Assoc 2002;41:283-289.

23) DuPaul GJ, Power TJ, McCoey KE, Ikeda MJ, Anastopoulos AD. Reliability and validity of parent and teacher rating of attention-deficit/hyperactivity disorder symptoms. J Psychoeduc Assess 1998;16: 55-68.

24) Murphy KR, Adler LA. Assessing attention-deficit/hyperactivity disorder in adults: focus on rating scales. J Clin Psychiatry 2004;65 Suppl 3:12-7.

25) World Health Organization. Adult ADHD Self-Report Scale-vl.1 (ASRS-v1.1) symptom checklist from WHO composite international diagnostic interview. Geneva: World Health Organization;2003.

26) Adler LA, Spencer T, Faraone SV, Kessler RC, Howes MJ, Biederman J, et al. Validity of pilot Adult ADHD Self- Report Scale (ASRS) to Rate Adult ADHD symptoms. Ann Clin Psychiatry 2006;18:145-148. 
M Kim, et al.

27) Green JG, DeYoung G, Wogan ME, Wolf EJ, Lane KL, Adler LA. Evidence for the reliability and preliminary validity of the Adult ADHD Self-Report Scale v1.1 (ASRS v1.1) Screener in an adolescent community sample. Int J Methods Psychiatr Res 2019;28:e1751.

28) Brandt VC, Beck C, Sajin V, Anders S, Münchau A. Convergent Validity of the PUTS. Front Psychiatry 2016;7:51. 